\title{
Suppression of Glow Corona on Streamer and Influence of Thin Wire on its Inception
}

\author{
Wenxia Sima*, Shuochao Fan ${ }^{\dagger}$, Qing Yang* and Qi Wang*
}

\begin{abstract}
Glow corona is a kind of streamer-free corona that can suppress upward leaders in transmission lines. Thus, it has good application potential in lightning protection. This paper investigates its corona characteristics. The suppression characteristic of glow corona on streamer is studied in air gap under negative DC voltage by wrapping thin wires on the electrode. The effect of thin wire winding patterns on the gap breakdown voltage is analyzed. Results are considered to be attributed to the inception condition of glow corona. Thus an inception test of glow corona is also conducted, and the inception voltage is obtained. Results show that the inception voltage decreases with short winding pitch. Thus an investigation on the inception of glow corona influenced by thin wire is conducted, and an influential factor is proposed to evaluate the influence. The inception regular of thin wire glow corona presented in this paper has certain reference value for the application of glow corona in transmission lines.
\end{abstract}

Keywords: Thin wire, Glow corona, Streamer suppression, Corona inception

\section{Introduction}

Lightning discharge in nature is a long gap discharge. A charge center exists at the bottom of thundercloud, and a large approximate capacitor appears between the thundercloud and the ground. At some part of the negative charge center,when the atmospheric electric field reaches a magnitude of $10 \mathrm{kV} / \mathrm{m}$, the initial breakdown process involves the breakdown of atmosphere and the appearance of streamers occurs. Then, a dark-light pillar gradually extends to the ground, which is known as a stepped leader, and a descending lightning diacharge channel forms. The electric field around the object on the ground increases with the development of descending lightning leader. The upward leader initiates when the electric field increases to a certain degree. And a lightning strike occurs when the descending and upward leaders attract each other and meet. Therefore, the essence of lightning strike is that the lightning leader hits the upward leader from the ground object but not the object itself. Hence, suppressing the formation and development of upward leaders from power conductors in transmission lines may be an effective way to reduce the probability of lightning strikes on power conductors. Many experiments and data [1-2] show that the presence of an approaching positive streamer of critical size is a prerequisite for continuous positive leader formation. Therefore streamer inhibition will has good effect on

$\dagger$ Corresponding Author: State Key Laboratory of Power Transmission Equipment \& System Security and New Technology, Chongqing University, Chongqing, China. (fanshuochao@cqu.edu.cn)

* State Key Laboratory of Power Transmission Equipment \& System Security and New Technology, Chongqing University, Chongqing, China. (cqsmwx@cqu.edu.cn)

Received: May 7, 2014; Accepted: January 23, 2015 preventing the formation of upward leaders.

As the thundercloud electric field varies slowly, a streamer-free corona (glow corona) generates from the grounded object. As a result, the glow corona could suppress the development of streamer and then inhibit the generation of upward leader [3-8]. Uhlig [9] conducted a study on glow corona and thought that the ideal electrode form for generating glow corona is thin wire. Then, some scholars attempted to apply thin wires to power conductors in transmission lines. Popkove [10] studied the radio interference characteristics of a high-voltage, direct current transmission line by wrapping $2 \mathrm{~mm}$ diameter spiral wires on a three-bundle conductor which is installed $2.6 \mathrm{~m}$ above the ground plane. The study showed that the application of thin wire changes the corona mode from streamer to glow by suppressing streamer formation. Heroux [11] conducted a test on improving the corona performance of a high-voltage conductor and concluded that the ionic space charges from glow corona lower the electric field at the conductor surface and thus suppress corona activity efficiently. However, the application of thin wire on power conductors is not advised because of the great corona losses. On the basis of previous researches, Rizk [12] wrapped different types of thin wires on the electrodes of air gap and measured the corona current, charges, and breakdown voltage under dry and wet conditions. Basing on theoretical analysis [13], he suggested wrapping thin wires on ground wires in transmission lines to produce glow corona and suppress the upward leader from power conductors and then improve the lightning protection performance.

To date, only a few studies have explained the application potential of glow corona in lightning protection of 
transmission lines. But no one has investigated the corona characteristics of glow corona in detail, which is the application foundation of glow corona. Therefore, the present study used previous works as basis to investigate the corona characteristics in detail. An experiment was conducted to test the streamer inhibitory effect of glow corona in air gap under positive DC voltage. An analysis on the influence of thin wire wrapping types on breakdown voltage is conducted. A test on the inception of thin wire glow corona is carried out, and the inception voltage is obtained. An investigation on the inception regular of glow corona influenced by thin wire is conducted. The inception regular of thin wire glow corona presented in this paper has certain reference value for the application of glow corona in transmission lines.

\section{Experimental Study on Suppression Characteristic on Streamer of Thin Wire Ultra Corona}

Air gap is broken down from corona inception to streamer development. Gap breakdown occurs when the gap is bridged by the streamer (or even leader). This bridged process cannot be achieved easily if the existence of glow corona can suppress the streamer development to improve the breakdown voltage of the gap. Therefore, the suppression effect of glow corona on streamer can be analyzed by the breakdown test of air gap.

\subsection{Selection of electrode types}

The streamer inhibitory effect of thin wire glow corona relies on the stability itself under the varied voltages. Only a stable corona can effectively inhibit the streamer. When in an infinitesimal time interval $\triangle t$, the glow corona should generate a sufficient number of positive ions escaping from the electrode to remain stable, so that the maximum electric field does not exceed the corona inception field $E_{c i}$ [12]. Thus, the rate of voltage rise has a critical maximum value.

In this study, the high-voltage electrode is a circular plate electrode to provide a slowly varied electric field, like a thundercloud electric field. The ideal electrode type for generating glow corona is thin wire [9]. However, thin wires cannot be used directly as grounding electrode because of their fragility. In this study, thin wires are wrapped to other grounding electrode type to generate glow corona while ensuring the mechanical property of the grounding electrode. Hence, the grounding electrode should be easily wrapped with thin wire and capable of stabilizing the glow corona. Rizk has provided the critical stability criterion of glow corona for spherical, toroidal, and cylindrical electrodes [12], which are as follows, respectively.

$$
\begin{gathered}
M_{1} \leq 2 \mu E_{c i}^{2} \\
M_{2} \leq\left[1+G(c)+c \frac{d G}{d c}\right] \mu E_{c i}^{2} \\
M_{3} \leq \mu \cdot \ln \frac{2 H}{R} \cdot E_{c i}^{2}
\end{gathered}
$$

where $M$ is the rate of voltage rise; $\mu$ is the positive ion mobility, $\mu=1.95 \times 10^{-4} \mathrm{~m}^{2} / \mathrm{V} \cdot \mathrm{s}$ [14]; $c=r / b, r$ and $b$ are major radius and minor radius of toroidal electrode; $G(c)=K(m$, $\pi / 2), K$ is the complete elliptic integral of the first kind, $m^{2}=1-(r / 2 b)^{2} ; H$ is the height of cylindrical electrode and $R$ is the electrode radius.

The minimal value in free space of $\left[1+G(c)+c \frac{d G}{d c}\right]$ is

5.77 [12]. The value of $\ln (2 H / R)$ is 4.61 according to the arrangement parameters of this paper in laboratory. Therefore, the value of $M_{2}$ for toroidal electrode is the maximum and toroidal electrode has the superiority of maintaining the stability of glow corona. That's why Rizk chose toroidal electrode as the grounding electrode in his research. However, we find that the value of $M_{3}$ is not only related to the dimension of the electrode itself, but also related to the height $H$. It is known that glow corona has application potential in transmission lines. So the height may be several times higher than that in laboratory, and then the value of $M_{3}$ can be much greater. Meanwhile, the application mode of glow corona is wrapping thin wires on ground wire [13]. The ground wire in transmission line is a kind of electrode type similar to cylindrical electrode. Therefore, more attentions are paid on how is the streamer inhibitory effect of cylindrical electrode in this paper, and the experiment on the suppression characteristic of glow corona on streamer will adopt this electrode type as grounding electrode.

\subsection{Arrangement of equipment}

The sketch of test circuit is shown in Fig. 1. The rated capacity and current of testing transformer are $300 \mathrm{kVA}$ and $1 \mathrm{~A}$. The output voltage is up to $300 \mathrm{kV}$, and the voltage

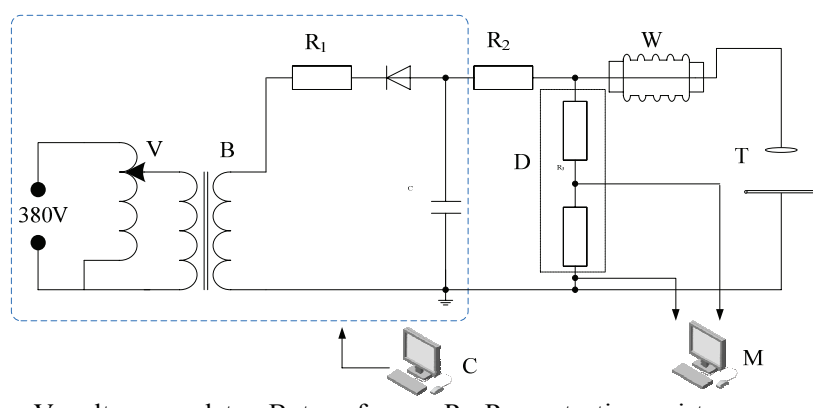

$\mathrm{V}$ : voltage regulator, $\mathrm{B}$ : transformer, $\mathrm{R}_{1}, \mathrm{R}_{2}$ : protective resistor,

$\mathrm{D}$ : direct voltage divider, $\mathrm{W}$ : wall bushing, $\mathrm{T}$ : testing air gap and electrodes, C: controlling system, M: measuring system.

Fig. 1. Sketch of the test circuit 
ripple factor is less than $3 \%$ when rated outputing. Protective resistors $R_{l}$ and $R_{2}$ are added to avoid excessive testing current, where $R_{l}=5 \mathrm{k} \Omega$ and $R_{2}$ is a pure water resistance with conductivity less than $2 \mu \mathrm{S} / \mathrm{cm}$. The highvoltage electrode is a circular plate electrode with a $60 \mathrm{~cm}$ diameter to provide a slowly varied electric field. The grounding electrodes is cylindrical (diameter: $1 \mathrm{~cm}$ ), which is wrapped with thin wires. The thin wire is stainless steel fiber with $60 \mu \mathrm{m}$ diameter, which has well electrical conductivity, soundness, and thermostability. The thin wires are wrapped around the electrode to form a coil.

In the test, negative polarity voltage is applied to the high-voltage electrode and rises uniformly until the gap breakdown at a rate of $0.5 \mathrm{kV} / \mathrm{s}$. The production degree and effect of glow corona can be controlled by changing the winding pitch of thin wires to compare the suppression effect on the streamer and the influence on the breakdown voltage.

\subsection{Results and analysis}

The breakdown voltages under different winding pitches and gap distances are obtained from the test and the results are shown in Fig. 2. The figure shows that the breakdown voltage of air gap increases after wrapping thin wires. For the breakdown voltage of thin wire wrapped electrode, the dispersion of the voltages decreases with the increase of gap length. The reason is that when the gap length is short, sometimes the streamer is not suppressed adequately by glow corona. Then the gap has been bridged by streamer, which leads to a low breakdown voltage less than the average value. And when the gap length is quite long, streamer is fully suppressed by glow corona. So the

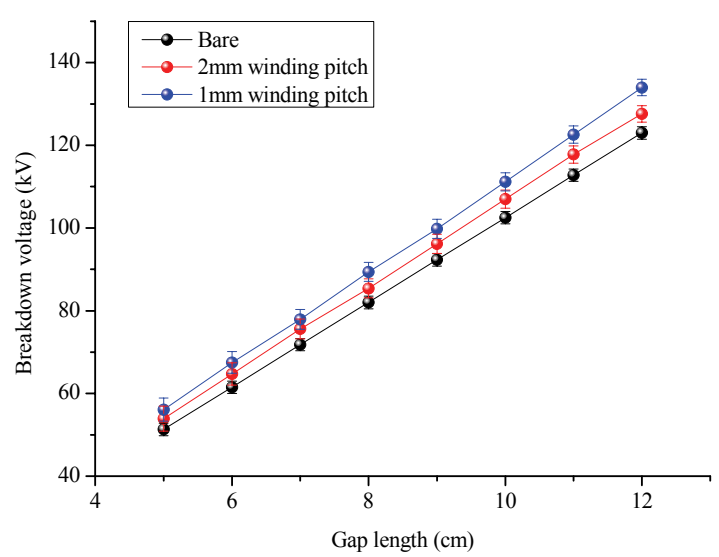

Fig. 2. Breakdown voltages of cylindrical electrode under different winding pitches and gap distances

Table 1. Increase ratio of breakdown electric field strength of cylindrical electrode

\begin{tabular}{c|c}
\hline Cladding & Increase ratio \\
\hline Bare & - \\
\hline $2 \mathrm{~mm}$ pitch & $3.4 \%$ \\
\hline $1 \mathrm{~mm}$ pitch & $8.7 \%$ \\
\hline
\end{tabular}

breakdown voltage is close to the average value. The average values of breakdown electric fields under different winding pitches are calculated and the increase ratios are shown in Table 1.

The above results demonstrate that the breakdown voltage and electric field both increase after the electrode is wrapped with thin wires. The increase in percentage is greater with a shorter winding pitch. For the cylindrical electrode, the formation of glow corona by wrapping thin wires suppresses the development of streamer and makes the gap channel not easily be bridged by it. As a result, the breakdown voltage and field increase. The streamer-free space charges produced by glow corona increase with shorter winding pitch, making the suppression effect better. Furthermore, the cylindrical electrode types also has superiority on maintaining the long-term stability of glow corona. As a result, the breakdown voltage increases.

\section{Study on Inception Regular of Thin Wire Glow Corona}

For cylindrical electrode, the suppression effect is better when the winding pitch of thin wires is shorter and the breakdown voltage is higher. This result is attributed to a matter of inception time of glow corona. A glow corona that incepts earlier produces more streamer-free space charges and makes the development of glow corona last longer. Thus, the suppression effect on streamer is better. Therefore, the relationship between the wrapping type of thin wire and the inception regular of glow corona will be studied through the inception experiment of glow corona to obtain the influence of thin wire on the inception of glow corona. If thin wire is applied to the ground wire of transmission lines, this influence will certainly affect the application effect of glow corona. The analysis in this section could have certain reference value for the application of glow corona in transmission lines.

\subsection{Test on corona inception and result analysis}

Refering to the inception measurement circuit in [12], the test circuit in Section 2 is redesigned (Fig. 3). The high voltage part is a circular plate electrode of $60 \mathrm{~cm}$ diameter to provide a slowly varied electric field. The grounding electrode is a cylindrical electrode with a diameter of $1 \mathrm{~cm}$. The grounding electrode is wrapped with thin wires in varying winding pitches. The distance between the two electrodes is $5 \mathrm{~cm}$, and the grounding electrode is connected to the earth through a sampling current sensor. This experiment is conducted in the electromagnetic shield room of Chongqing University, and the various interferences can be reduced effectively to ensure the accuracy of the test data. The ambient temperature is $300 \mathrm{~K}$, and the background atmospheric pressure is $1 \mathrm{~atm}$.

In the experiment, negative polarity voltage is applied to 


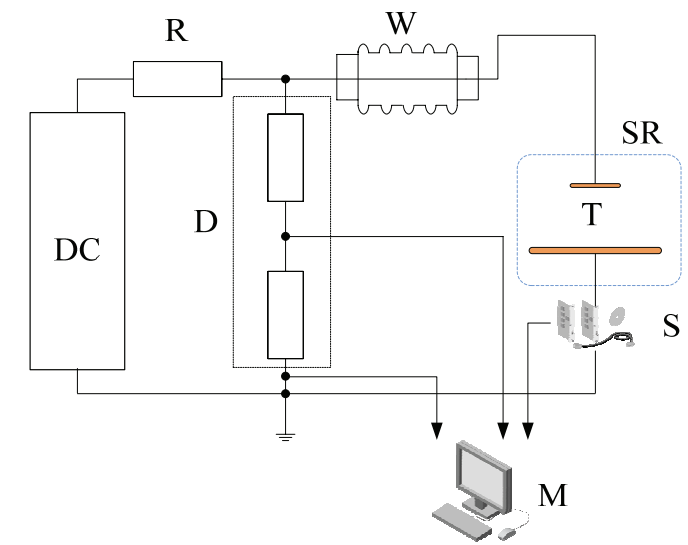

DC: DC high voltage, R: damping resistor, D: direct voltage divider, W: wall bushing, SR: electromagnetic shield room, T: testing air gap and electrodes, S: current sensor, M: measuring system.

Fig. 3 Schematic of the test circuit

Table 2. Corona inception voltages with different winding pitches

\begin{tabular}{c|c}
\hline Cladding & Inception voltage $(\mathrm{kV})$ \\
\hline Bare & 48.9 \\
\hline 4 mm pitch & 45.6 \\
\hline 3 mm pitch & 44.2 \\
\hline 2 mm pitch & 41.7 \\
\hline 1 mm pitch & 35.5 \\
\hline
\end{tabular}

the high-voltage electrode by the DC power supply and rises uniformly at a rate of $0.1 \mathrm{kV} / \mathrm{s}$. Glow corona is judged to incept by the start of discharge current flow (sensitivity $\approx 10 \mu \mathrm{A}[12]$ ), and then the voltage is recorded.

Table 2 shows the experiment result. The inception voltage of glow corona decreases with a shorter winding pitch. This is due to the difference of the electric field distribution on surface between smooth conductor and thin wire-wrapped conductor. The surface density of charges is inversely proportional to curvature radius. If a point is wrapped with thin wire, the curvature radius on its surface is changed to be far smaller than that of a smooth point. Then, the surface density of charges at this bulgy point is also far greater than that of a smooth point, so is the electric field. Thus, the increased field can easily reach the required strength for corona inception. The amount of bulgy points increases as the winding pitch becomes shorter, so that more points on the surface could meet the inception condition before the applied voltage increases to the inception value of smooth conductor. Thus, the inception voltage becomes lower.

\subsection{Influence of thin wires on the inception regular of glow corona}

If the conductor is wrapped with thin wires, the surface is no longer smooth. Namely, wrapping thin wires changes the inception regular of the corona. Because the radius of thin wires is too small, wrapping thin wires to the conductor has very little impact on the diameter of the conductor. Thus the diameter of the conductor is considered to be unchanged. Then the influence of thin wire on the inception of glow corona is related to two factors. One is the influence from the thin wire diameter, because it can change the curvature radius of the bulgy points on the surface, the other is the coverage area (or winding pitch) on the conductor of thin wires, which determines the amount of bulgy points on the surface. Therefore, to make a deep analysis on the influence of thin wires on the inception regular of glow corona, a closeness density factor $k\left(=d_{0} / d\right)$ is presented to reflect the influence, where $d_{0}$ is the diameter of thin wire and $d$ is the winding pitch. In addition, the influential factor of wrapping thin wire is defined as

$$
m=\frac{U_{c m}}{U_{c 0}}
$$

where $U_{c m}$ is the inception voltage of thin wire-wrapping conductor and $U_{c 0}$ is the inception voltage of smooth conductor. A small value of $m$ means greater influence of thin wire on the corona inception.

According to the inception voltages of varying winding pitches in Table 2, the values of $m$ with different $k$ are calculated, and the expression of $m$ is fitted. The best fitting curve follows negative exponential function.

$$
m=m_{0} e^{-a k}
$$

where $m_{0}$ and a are constants; $k$ is the closeness density factor of thin wire wrapping. The fitting curve is shown in Fig. 4.

According to the fitting result, the expression of $m$ is

$$
m=e^{-5.344 k}
$$

The formula shows that the influential factor $m$ decreases as $k$ increases. Then the influence of thin wires on the surface density of charges becomes greater and the inception of glow corona becomes easier. Earlier glow

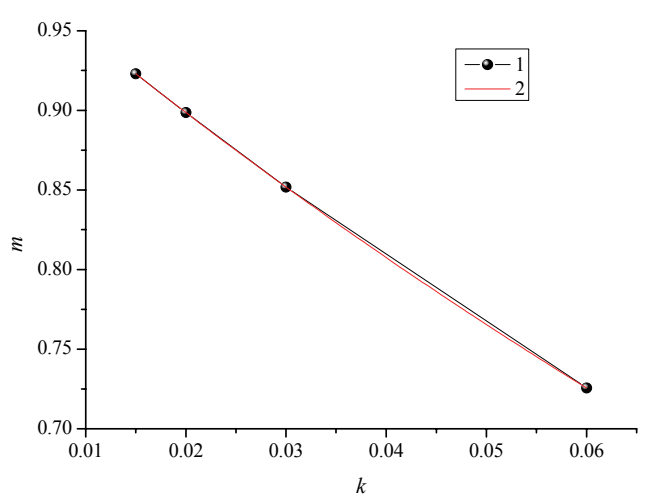

Fig. 4. The value of $m$ under different $k$ 


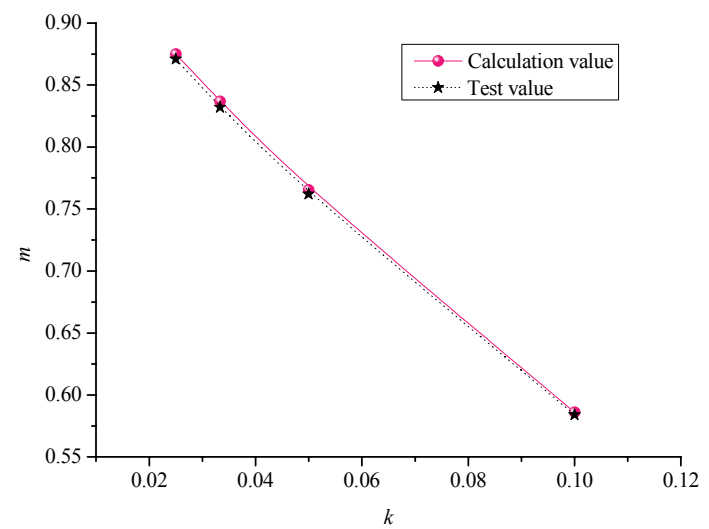

Fig. 5. Comparison of test value and calculation value of $m$ with different winding pitches

corona inception means more streamer-free space charges are produced; thus, the suppression effect on streamer becomes stronger. Hence, the supressed streamer develops slowly and the whole gap is difficult to be bridged, resulting in an improved breakdown voltage or electric field. This analyzed result concides with the test results in Section 2.3, and it is the theoretical verification.

To verify the universality of (6), the diameter of thin wire is changed to $100 \mu \mathrm{m}$, and the diameter of the conductor is changed to $2 \mathrm{~cm}$. The corona inception experiment in this section is repeated, and the value of $m$ is obtained (named as test value). At the same time, the calculation value is also calculated through (6). The comparison of two values in different pitches is shown in Fig. 5, which shows that the two curves coincide well.

The influential factor $m$ proposed in this paper reflects the influence of thin wires on the inception regular of glow corona. Since glow corona may be applied to transmission lines in the furture and different wrapping patterns of thin wires really influence the inception process of glow corona actually, the investigation in this paper could have certain reference value for the application of glow corona in transmission lines.

\section{Conclusions}

This paper investigates the suppression of glow corona on streamer and its inception regular influenced by wrapping thin wires. The conclusions are as follows:

(1) Glow corona could suppress the development of streamer, making the streamer not easy to fill throughout the whole air gap. As a result, the breakdown voltage and electric field both improve. The amount of streamerfree space charges from glow corona increases and the suppression effect significantly improves as the winding pitch becomes shorter.

(2) The cylindrical electrode has superiority on maintaining glow corona stable, especially when applied to the conductors with high height.

(3) After wrapping thin wire to the conductor, the distribution of surface charges is changed. An influential factor $m$ is proposed in this paper to reflect the influence of thin wires on the inception regular of glow corona. Results show that the inception voltage decreases with smaller $m$.

(4) If glow corona is applied to transmission lines for lightning protection by wrapping thin wires to ground wire, the investigation in this paper could have certain reference value for it.

\section{References}

[1] Les Renardières Group, "Long air gap discharges at les renardières: 1973 results", Electra, no. 23, pp. 53157, Jul. 1972 and no. 35, pp. 49-156, Jul, 1974.

[2] F. A. M. Rizk, "A model for switching impulse leader inception and breakdown of long air gaps", IEEE Trans. on Power Del., vol. 4, no. 1, pp. 596-606, Jan. 1989.

[3] M. S. Mokrov, Y. P. Raizer, E. M. Bazelyan, "Development of a positive corona from a long grounded wire in a growing thunderstorm field", J. Phys. D., vol. 46, 455202, Oct. 2013.

[4] N. L. Aleksandrov, E. M. Bazelyan, Y. P. Raizer, "The effect of corona discharge on lightning attachment," Plasma Phys. Rep., vol. 31, no. 1, pp. 75-91, 2005.

[5] N. L. Aleksandrov, E. M. Bazelyan, R. B. Carpenter, M. M. Drabkin, Y. P. Raizer, "The effect of coronae on leader initiation and development under thunderstorm conditions and in long air Gaps," J. Phys. D.: Appl. Phys., vol. 34, pp. 3356-3266, 2001.

[6] N. L. Aleksandrov, E. M. Bazelyan, M. M. Drabkin, R. B. Carpenter, Y. P. Raizer, "Corona discharge at the tip of a tall object in the electric field of a thundercloud," Plasma Phys. Rep., vol. 28, no. 11, pp. 953 964, 2002.

[7] E. M. Bazelyan, N. L. Aleksandrov, F. D’Alessandro, Y. P. Raizer, "Numerical simulation of thunderstorm induced processes near lightning rods installed on grounded structures," in Proc. 28th Int. Conf. Lightning Protection, 2006, pp. 564-569.

[8] E. M. Bazelyan, M. M. Drabkin, "Scientific and technical basis for preventing lightning strikes to earthbound objects", Power Engineering Society General Meeting (Toronto, 13-17 July 2003) vol. 4 (Piscataway, NJ: IEEE), pp. 2201-8, 2003.

[9] C. A. E. Uhlig, "The ultra corona discharge, a new phenomenon occurring on thin wires", presented at the High Voltage Symposium, Nat. Res. Council Canada, Ottawa, ON, Canada, 1956.

[10] V. I. Popkov, "Some special features of corona on high voltage DC transmission lines", in Gas Dis- 
charges and the Electricity Supply Industry Paper no. 38. London, U.K.: Butterworths, 1962, pp. 225-237.

[11] P. Heroux, P.S. Maruvada, N.G. Trinh, "High voltage AC transmission lines: Reduction of corona under foul weather", IEEE Trans. Power App. Syst., vol. PAS-101, no. 9, pp. 3009-3017, Sep. 1982.

[12] F. A. M. Rizk, "Analysis of space charge generating devices for lightning protection: Performance in slow varying fields", IEEE Trans Power Del., vol. 23, no. 3, pp. 1996-2006, Jul. 2010.

[13] F. A. M. Rizk, "Exposure of overhead conductors to direct lightning strikes: Modeling of positive streamer inhibition", IEEE Trans. Power Del., vol. 26, no. 2, pp. 1156-1165, Apr. 2011.

[14] Y. Higashiyama, K. Nagaki, K. Yatzuka, "Measurement of positive ion mobility in air using pulsed corona discharge", Trans., Inst. Elect. Eng. Jpn., vol. 121-a, no. 11, pp. 984-989. 2001.

[15] E. M. Bazelyan, Y. P. Raizer, N. L. Aleksandrov, "Corona initiated from grounded objects under thunderstorm conditions and its influence on lightning attachment", Plasma Sources Science and Technology., vol. 17, 024015, 2008.

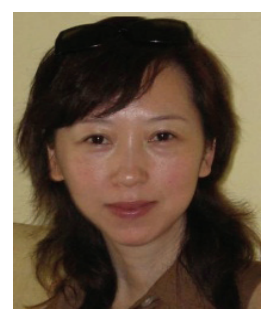

Wenxia Sima She was born in Henan province, China, on 13 July 1965. She graduated from Chongqing University in 1988 and obtained the Ph.D. degree in 1994 from Chongqing University. Her employment experience includes the college of Electrical Engineering of Chonging University. Her fields of interest include high voltage outdoor insulation and overvoltage protection of transmission and distribution apparatuses.

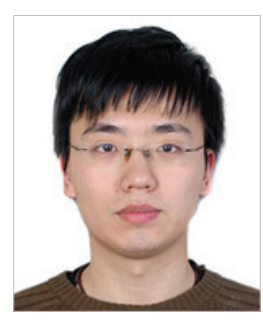

Shuochao Fan He was born in Hebei province, China, on November, 1987. He received the B.S. degree from Chongqing University in 2010. He is now a Ph.D. candidate in the State Key Laboratory of Power Transmission Equipment \& System Security and New Technology, Chongqing University. His major research interest is lightning protection of transmission lines.

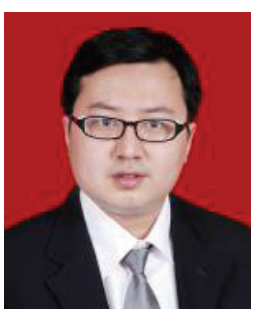

Qing Yang He received the B.S and Ph.D. degrees in electrical engineering, respectively, in 2002 from North China Electrical Power University and in 2006 from Chongqing University, China. $\mathrm{He}$ is now an Associate Professor in the State Key Laboratory of Power Transmission Equipment \& System Security and New Technology, Chongqing University. His research interests include outdoor insulation in complex ambient conditions and electric-field calculations.

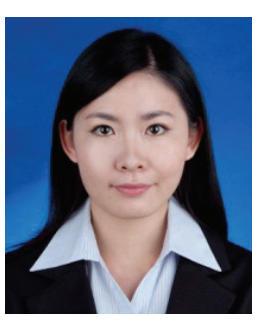

Qi Wang She was born in Yichang, Hubei Province, China, on June, 1990. She received the B.S. degree at China Three Gorges University in 2012. She is now a Master. candidate in the State Key Laboratory of Power Transmission Equipment \& System Security and New Technology, Chongqing University. Her major research interest is lightning protection of transmission lines. 